\title{
Analytical Study on Unsteady MHD Free Convection and Mass Transfer Flow Past a Vertical Porous Plate
}

\author{
S. F. Ahmmed, M. K. Das, L. E. Ali \\ Mathematics Discipline, Khulna University, Khulna-9208, Bangladesh
}

Email address:

sfahmmed@yahoo.com (S. F. Ahmmed),mahadeb.ku@gmail.com (M. K. Das), liponmath@yahoo.com (L. E. Ali)

To cite this article:

S. F. Ahmmed, M. K. Das, L. E. Ali. Analytical Study on Unsteady MHD Free Convection and Mass Transfer Flow Past a Vertical Porous Plate. American Journal of Applied Mathematics. Vol. 3, No. 2, 2015, pp. 64-74. doi: 10.11648/j.ajam.20150302.16

\begin{abstract}
The unsteady MHD free convection and mass transfer boundary layer flow past a semi-infinite vertical porous plate immersed in a porous medium with heat source is studied. The plate moves with a uniform velocity in the direction of the fluid flow while the free stream velocity is considered to follow the exponentially increasing small perturbation law. The governing equations are solved analytical by using perturbation technique depending on the physical parameters including the Radiation parameter $(R)$, the Magnetic parameter $(M)$, the Prandtl number $(P r)$, the Grashof number for heat transfer $(G r)$, the Modified Grashof number for mass transfer $(G c)$, the Schmidt number $(S c)$, the soret number $\left(S_{0}\right)$, the permeability parameter $(K)$ and the heat source $(Q)$. The influence of these parameters on velocity field, temperature field, concentration field, skin friction, Nusselt number and Sherwood number at the plate are discussed with graphically.
\end{abstract}

Keywords: MHD, Heat and Mass Transfer, Porous Plate, Perturbation Law, Heat Source, Soret Number

\section{Introduction}

The study of unsteady MHD free convection flow with mass transfer along a vertical porous plate is receiving considerable attention of many researchers because of its various applications. Permeable porous plates are used in the filtration processes and also for a heated body to keep its temperature constant and to make the heat insulation of the surface more effective. Sometimes along with the free convection currents caused by difference in temperature the flow is also affected by the differences in concentration or material constitution. This type of flow has applications in many branches of science and engineering. The study of such flow under the influence of magnetic field has attracted the investigators in view of its various applications in MHD generators, plasma studies, nuclear reactors, geothermal energy extractions and boundary layer control in the field of aerodynamics. Moreover, considerable interest has been shown in radiation interaction with convection for heat and mass transfer in fluids. This is due to the significant role of thermal radiation in the surface heat transfer when convection heat transfer is small, particularly in free convection problems involving absorbing-emitting fluids.

Several workers have studied the problem of free convection flow with mass transfer. Singh et al. [1] have studied MHD free convective flow past an accelerated vertical porous plate by finite difference method. Free convection and mass transfer flow through porous medium bounded by an infinite vertical limiting surface with constant suction have been analyzed by Raptis et al [2]. Unsteady free convection interaction with thermal radiation in a boundary layer flow past a vertical porous plate has been discussed by Sattar et al [3]. Das et al [4] have studied numerical solution of mass transfer effects on unsteady flow past an accelerated vertical porous plate with suction. Das et al [5] have studied Mass transfer effects on MHD flow and heat transfer past a vertical porous plate through porous medium under oscillatory suction and heat source. Applied magnetic field on transient convective flow in a vertical channel has been discussed by Jah [6].Kim [7] has investigated the problem of unsteady MHD convective heat transfer past a semi-infinite vertical porous moving plate with variable suction. Soundalgekar et al. [8] have analyzed the transient free convection flow of a viscous dissipative fluid past a semiinfinite vertical plate. Mohameda et al [9] have analyzed finite element analysis of hydromagnetic flow and heat transfer of a heat generation fluid over a surface embedded in a non-darcian porous medium in the presence of chemical reaction. The Soret effect on free convective unsteady MHD flow over a vertical plate with heat source has been analyzed by Bhavana et al. [10]. Abd EL-Naby et al [11] employed 
implicit finite finite-difference methods to study the effect of radiation on MHD unsteady free convection flow past a semi-infinite vertical porous plate but did not take into account the viscous dissipation.

Recently, Alam and Rahman [12] have examined Dufour and Soret effects on mixed convection flow past a vertical porous flat plate with variable suction embedded in a porous medium for a hydrogen-air mixture as the nonchemical reacting fluid pair. Anwa. et al. [13] examined the combined effects of Soret and Dufour diffusion and porous impedance on laminar magneto-hydrodynamic mixed convection heat and mass transfer of an electrically-conducting, Newtonian, Boussinesq fluid from a vertical stretching surface in a Darcian porous medium under uniform transverse magnetic field. Hady et al. [14] studied the problem of free convection flow along a vertical wavy surface embedded in electrically conducting fluid saturated porous media in the presence of internal heat generation or absorption effect. Makinde [15] have discussed Free-convection flow with thermal radiation and mass transfer past a moving vertical porous plate.

From the previous literature survey about unsteady fluid flow, we observe that a few papers have done on porous medium. The effect of radiation on MHD flow and heat transfer must be considered when high temperatures are reached. The study of heat generation or absorption effects in moving fluids is important in view of several physical problems, such as fluid undergoing exothermic or endothermic chemical reactions.

When the mass flux contains a term that depends on the temperature gradient then the soret effect arises. The major focus of our study is the effect on free convection of the addition of a second fluid. Convection in binary fluids is considerably more complicated than that in pure fluids. Both temperature and concentration gradients contribute to the initiation of convection and each may be stabilizing or destabilizing. Even when a concentration gradient is not externally imposed (the thermosolutal problem) it can be created by the applied thermal gradient via the soret effect. Mbeledogu et.al [16] have discussed the unsteady MHD free convection flow of a compressible fluid past a moving vertical plate in the presence of radioactive heat transfer. Ahmmed et al. [17] have discussed Numerical Study on MHD free convection and mass transfer flow past a vertical flat plate.

In our present paper the unsteady MHD free convection and mass transfer flow past a vertical porous plate has been investigated analytically by using perturbation technique. The effects of the flow parameters on the velocity, temperature, concentration, skin friction, Nusselt number and Sherwood number distribution of the flow field have been studied with the help of graphs.

\section{Formulation of the Problem}

Consider a two dimensional unsteady flow of a laminar, incompressible, viscous, electrically conducting and heat generation fluid past a semi-infinite vertical moving plate embedded in a uniform porous medium and subjected to a uniform transverse magnetic field in the presence of a pressure gradient has been considered with free convection, thermal diffusion and thermal radiation effects taking in to an account. According to the coordinate system the $x^{*}$-axis is taken along the porous plate in the upward direction and $y$ *axis normal to it. The fluid is assumed to be gray, absorbing-emitting but not scattering medium. The radiative heat flux in the $x^{*}$-direction is considered negligible in comparison with that in the $y^{*}$-direction. It is assumed that there is no applied voltage of which implies the absence of an electric field. The transversely applied magnetic field and magnetic Reynolds number are very small and the induced magnetic field is negligible. Viscous and Darcy resistance terms are taken into account the constant permeability porous medium. The MHD term is derived from an order of magnitude analysis of the Navier-stokes equation. The fluid properties are considered to be constants except that the influence of density variation with temperature and concentration has been assumed in the body-force term. Due to the semi-infinite plate surface assumption, the flow variable are functions of $y^{*}$ and $t^{*}$ only. The governing equation for this observation is based on the balances of mass, linear momentum, energy and concentration species.

Within the frame work of delete such assumptions the equations of continuity, momentum, energy and concentration are follows, Bhavana et al. [10]

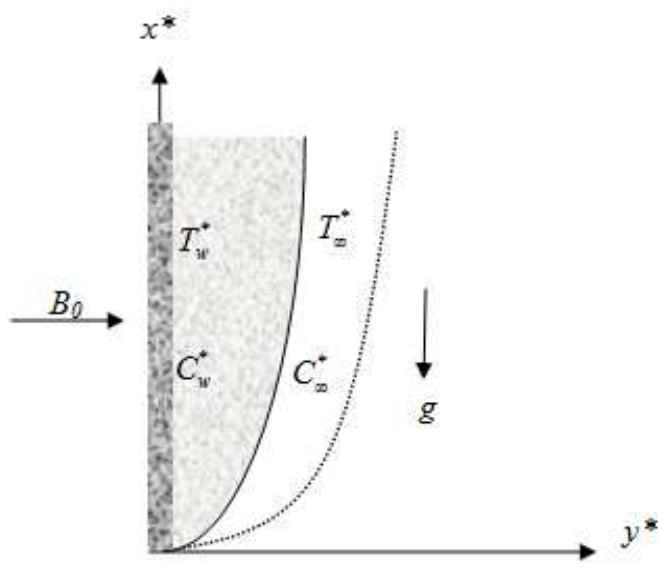

Figure 1. Physical model of boundary layer.

$$
\begin{gathered}
\frac{\partial v^{*}}{\partial y^{*}}=0 \\
\rho\left(\frac{\partial u^{*}}{\partial t^{*}}+v^{*} \frac{\partial u^{*}}{\partial y^{*}}\right)=\frac{\partial p^{*}}{\partial x^{*}}+\mu \frac{\partial^{2} u^{*}}{\partial y^{* 2}}-\rho \beta-\frac{\mu}{K^{*}} u^{*}-\sigma B_{0}{ }^{2} u^{*} \\
\frac{\partial T^{*}}{\partial t^{*}}+v^{*} \frac{\partial T^{*}}{\partial y^{*}}=\frac{k}{\rho C_{p}} \frac{\partial^{2} T^{*}}{\partial y^{* 2}}-\frac{1}{\rho C_{p}}\left(\frac{\partial q_{r}^{*}}{\partial y^{*}}\right)-\frac{Q_{0}}{\rho C_{p}}\left(T^{*}-T_{\infty}^{*}\right) \\
\frac{\partial C^{*}}{\partial t^{*}}+v^{*} \frac{\partial C^{*}}{\partial y^{*}}=D_{M} \frac{\partial^{2} C^{*}}{\partial y^{* 2}}+D_{T} \frac{\partial^{2} T^{*}}{\partial y^{* 2}}
\end{gathered}
$$


where $x^{*}, y^{*}$ and $t^{*}$ are the dimensional distances along the plate, perpendicular to the plate and dimensional time, respectively. $u^{*}$ and $v^{*}$ are the components of dimensional velocities along $\mathrm{x}^{*}$ and $\mathrm{y}^{*}$ directions respectively, $\rho$ is the fluid density, $\mu$ is the velocity, $\mathrm{Cp}$ the specific heat at constant pressure, $\sigma$ is the fluid electrical conductivity, $B_{0}$ is the magnetic induction, $K^{*}$ is the permeability of the of the porous medium, $T^{*}$ is the dimensional temperature, $D_{M}$ is the coefficient of chemical molecular diffusivity, $D_{T}$ is the coefficient of thermal diffusivity, $C^{*}$ is the dimensional concentration is the thermal conductivity of the fluid, $g$ is the acceleration due to gravity and $q_{r}{ }_{r}$ and $R$ are the local radioactive heat flux and the reaction rate constant respectively.

The boundary conditions for the velocity, temperature and concentration fields are given as follows

$$
\begin{gathered}
u^{*}=u_{p}^{*}, T^{*}=T_{w}^{*}+\varepsilon\left(T_{w}^{*}-T_{\infty}^{*}\right) \mathrm{e}^{n^{*} t^{*}}, C^{*}=C_{w}^{*}+\varepsilon\left(\mathrm{C}_{w}{ }^{*}-C_{\infty}{ }^{*}\right) \mathrm{e}^{n^{*} t^{*}} \text { at } y^{*}=0 \\
u^{*} \rightarrow U_{\infty}^{*}=U_{0}\left(1+\varepsilon \mathrm{e}^{n^{*} t^{*}}\right), \quad \mathrm{T}^{*} \rightarrow T_{\infty}^{*}, \quad C^{*} \rightarrow C_{\infty}^{*} \quad \text { as } y^{*} \rightarrow \infty
\end{gathered}
$$

where $T_{w}{ }^{*}$ and $C_{w}{ }^{*}$ are the dimensional wall temperature and concentration respectively. $C_{\infty}{ }^{*}$ is the free stream dimensional concentration. $U_{0}$ and $n^{*}$ are constants.

From the equation (1), we consider the velocity as the exponential form

$$
v^{*}=-v_{0}\left(1+\varepsilon \mathrm{Ae}^{n^{*} t^{*}}\right)
$$

where, $A$ is the real positive constant, $\varepsilon$ and $\varepsilon A$ are small less than unity and $v_{0}$ is a scale of suction velocity which has non-zero positive constant.

In the free stream, we have

$$
\rho \frac{d U_{\infty}^{*}}{d t^{*}}=\frac{\partial p^{*}}{\partial x^{*}}-\rho_{\infty} g-\frac{\mu}{K^{*}} U_{\infty}^{*}-\sigma B_{0}^{2} U_{\infty}^{*}
$$

Eliminate $\frac{\partial p^{*}}{\partial x^{*}}$ using equation (2) and equation (8), we obtain

$$
\rho\left(\frac{\partial u^{*}}{\partial t^{*}}+v^{*} \frac{\partial u^{*}}{\partial y^{*}}\right)=\left(\rho_{\infty}-\rho\right) \mathrm{g}+\rho \frac{d \mathrm{U}_{\infty}^{*}}{d t^{*}}+\mu \frac{\partial^{2} u^{*}}{\partial y^{* 2}}-\frac{\mu}{K^{*}}\left(\mathrm{U}_{\infty}^{*}-u^{*}\right)-\sigma B_{0}{ }^{2}\left(\mathrm{U}_{\infty}^{*}-u^{*}\right)
$$

Also by using the equation of state, we have

$$
\left(\rho_{\infty}-\rho\right)=\beta\left(T^{*}-T_{\infty}^{*}\right)+\beta^{*}\left(C^{*}-C_{\infty}^{*}\right)
$$

Substituting equation (10) into equation (9), we have

$$
\frac{\partial u^{*}}{\partial t^{*}}+v^{*} \frac{\partial u^{*}}{\partial y^{*}}=\frac{d \mathrm{U}_{\infty}^{*}}{d t^{*}}+\mu \frac{\partial^{2} u^{*}}{\partial y^{* 2}}+g \beta\left(T^{*}-T_{\infty}^{*}\right)+g \beta^{*}\left(\mathrm{C}^{*}-C_{\infty}^{*}\right)+\frac{v}{K^{*}}\left(\mathrm{U}_{\infty}^{*}-u^{*}\right)+\frac{\sigma B_{0}{ }^{2}}{\rho}\left(\mathrm{U}_{\infty}^{*}-u^{*}\right)
$$

where, $v=\frac{\mu}{k}$ is the coefficient of the kinematic viscosity.

The radioactive heat flux term by using the Roseland approximation is given by

$$
q_{r}^{*}=\frac{4 \sigma^{*}}{3 k_{1}^{*}} \frac{\partial T^{*^{4}}}{\partial y^{*}}
$$

where, $\sigma^{*}$ and $k_{1}^{*}$ are respectively the Stefan-Boltzmann constant and the mean absorption coefficient.. We assume that the temperature difference within the flow are sufficiently small such that $T^{* 4}$ may be expressed as a linear function of the temperature. This is accomplished by expanding in a Taylor series about $T_{\infty}{ }^{*}$ and neglecting higher order terms, thus

$$
T^{* 4} \cong 4 T_{\infty}^{* 3}-3 T_{\infty}^{* 4}
$$

By using equation (12) and equation (13), into equation (3) is reduced to

$$
\frac{\partial T^{*}}{\partial t^{*}}+v^{*} \frac{\partial T^{*}}{\partial y^{*}}=\frac{k}{\rho C_{p}} \frac{\partial^{2} T^{*}}{\partial y^{* 2}}-\frac{16 \sigma^{*} T_{\infty}^{* 3}}{3 \rho C_{p} k_{1}^{*}} \frac{\partial^{2} T^{*}}{\partial y^{* 2}}-\frac{Q_{0}}{\rho C_{p}}\left(T^{*}-T_{\infty}^{*}\right)
$$

\section{Solution of the Problem}

To get the solution of the equation (1) to equation (4) with boundary conditions (5) and (6) we introduce the following non-dimensional quantities and parameters

$$
u^{*}=u U_{0}, v^{*}=v V_{0}, T^{*}=T_{\infty}^{*}+\theta\left(T_{w}^{*}-T_{\infty}^{*}\right), C^{*}=C_{\infty}^{*}+C\left(\mathrm{C}_{w}^{*}-C_{\infty}^{*}\right), \mathrm{U}_{\infty}^{*}=U_{\infty} U_{0}
$$




$$
\begin{gathered}
u_{p}{ }^{*}=U_{p} U_{0}, K^{*}=\frac{K v^{2}}{V_{0}{ }^{2}}, y^{*}=\frac{y v}{V_{0}}, G c=\frac{v g \beta^{*}\left(\mathrm{C}_{w}{ }^{*}-C_{\infty}{ }^{*}\right)}{V_{0}^{2} U_{0}}, G r=\frac{v g \beta\left(\mathrm{T}_{w}{ }^{*}-T_{\infty}{ }^{*}\right)}{V_{0}{ }^{2} U_{0}} \\
\operatorname{Pr}=\frac{v \rho C_{p}}{k}, M=\frac{\sigma B_{0}{ }^{2} v}{\rho V_{0}{ }^{2}}, Q=\frac{Q_{0} v}{\rho V_{0}{ }^{2} C_{p}}, R=\frac{4 \sigma^{*} T_{\infty}^{* 3}\left(\mathrm{~T}_{w}{ }^{*}-T_{\infty}{ }^{*}\right)}{k_{1}^{*} k}, S c=\frac{v}{D_{M}}, t^{*}=\frac{t v}{V_{0}^{2}}, n^{*}=\frac{V_{0}^{2}}{v}
\end{gathered}
$$

Therefore the governing equations in the dimensionless form become equation (16) to equation (19) with the boundary condition (20),

$$
\begin{gathered}
\frac{\partial v}{\partial y}=0 \\
\frac{\partial u}{\partial t}+v \frac{\partial u}{\partial y}=\frac{d U_{\infty}}{d t}+\frac{\partial^{2} u}{\partial y^{2}}+G r \theta+G m C+N\left(U_{\infty}-u\right) \\
\frac{\partial \theta}{\partial t}+v \frac{\partial \theta}{\partial y}=\frac{1}{P r}\left(1+\frac{4 R}{3}\right) \frac{\partial^{2} \theta}{\partial y^{2}}-Q \theta \\
\frac{\partial C}{\partial t}+v \frac{\partial C}{\partial y}=\frac{1}{S c} \frac{\partial^{2} C}{\partial y^{2}}+S_{0} \frac{\partial^{2} \theta}{\partial y^{2}}
\end{gathered}
$$

The corresponding initial and boundary conditions are

$$
\begin{array}{ll}
u=U_{p}, \theta=1+\varepsilon \mathrm{e}^{n t}, C=1+\varepsilon e^{n t} & \text { at } \quad y=0 \\
u \rightarrow U_{\infty} \rightarrow 1+\varepsilon \mathrm{e}^{n t}, \theta \rightarrow 0, C \rightarrow 0 & \text { as } \quad y \rightarrow \infty
\end{array}
$$

For perturbation technique to solve equation (16) to equation (19) we consider the solutions as the following forms

$$
\begin{aligned}
& u=u_{0}(y)+\varepsilon e^{n t} u_{1}(y)+0\left(\varepsilon^{2}\right) \\
& \theta=\theta_{0}(y)+\varepsilon e^{n t} \theta_{1}(y)+0\left(\varepsilon^{2}\right) \\
& C=C_{0}(y)+\varepsilon e^{n t} C_{1}(y)+0\left(\varepsilon^{2}\right)
\end{aligned}
$$

where, $u_{0}$ is mean velocity, $\theta_{0}$ mean temperature, $C_{0}$ mean concentration, $n$ is the frequency of the oscillation, $\varepsilon$ is a perturbation quantity and $t$ is the time respectively.

By substituting the above equations (21) into the equation

$$
\begin{aligned}
& u_{1}=1+J_{6} e^{m_{10} y}+J_{7} e^{m_{2} y}+J_{8} e^{m_{6} y}+J_{9} e^{m_{2} y}+J_{10} e^{m_{4} y}+J_{11} e^{m_{2} y}+J_{12} e^{m_{8} y}+J_{13} e^{m_{6} y}+J_{14} e^{m_{2} y}+J_{15} e^{m_{2} y}+J_{16} e^{m_{4} y}+J_{17} e^{m_{12} y} \\
& \theta_{0}=e^{m_{2} y} \\
& \theta_{1}=D_{1} e^{m_{2} y}+D_{2} e^{m_{4} y} \\
& C_{0}=B_{1} e^{m_{2} y}+B_{2} e^{m_{6} y} \\
& u(y, t)=1+J_{1} e^{m_{2} y}+J_{2} e^{m_{6} y}+J_{3} e^{m_{2} y}+J_{4} e^{m_{10} y}+\varepsilon e^{n t}\left(1+J_{6} e^{m_{10} y}+J_{7} e^{m_{2} y}+J_{8} e^{m_{6} y}+J_{9} e^{m_{2} y}+J_{10} e^{m_{4} y}\right. \\
& \left.+J_{11} e^{m_{2} y}+J_{12} e^{m_{8} y}+J_{13} e^{m_{6} y}+J_{14} e^{m_{2} y}+J_{15} e^{m_{2} y}+J_{16} e^{m_{4} y}+J_{17} e^{m_{12} y}\right) \\
& \theta(y, t)=e^{m_{2} y}+\varepsilon e^{n t}\left(D_{1} e^{m_{2} y}+D_{2} e^{m_{4} y}\right)
\end{aligned}
$$

(16) to equation (20), then equating the harmonic and nonharmonic terms and neglecting the higher order terms of $O\left(\varepsilon^{2}\right)$, we obtain the following pairs of equations for $\left(u_{0}, \theta_{0}\right.$, $\left.C_{0}\right)$ and $\left(u_{1}, \theta_{1}, C_{1}\right)$,

$$
\begin{gathered}
u_{0}^{\prime \prime}+u_{0}^{\prime}-N u_{0}=-N-G r \theta_{0}-G c C_{0} \\
u_{1}^{\prime \prime}+u_{1}^{\prime}-(N+n) u_{1}=-(N+n)-A u_{0}^{\prime}-G r \theta_{1}-G c C_{1} \\
(3+4 R) \theta_{0}^{\prime \prime}+3 \operatorname{Pr} \theta_{0}^{\prime}-3 \operatorname{Pr} \theta_{0}=0 \\
(3+4 R) \theta_{1}^{\prime \prime}+3 \operatorname{Pr} \theta_{1}^{\prime}-(3 n+Q) \operatorname{Pr} \theta_{1}=-3 A \operatorname{Pr} \theta_{0}^{\prime} \\
C_{0}^{\prime \prime}+S c C_{0}^{\prime}=-S_{0} S c \theta_{0}^{\prime \prime} \\
C_{1}^{\prime \prime}+S c C_{1}^{\prime}-n S c C_{1}=-A S c C_{0}^{\prime}-S_{0} S c \theta_{1}^{\prime \prime}
\end{gathered}
$$

where the primes denote differentiation with respect to $y$.

Then the corresponding boundary conditions can be written as

$$
\begin{array}{ll}
u_{0}=U_{p}, u_{1}=0, \theta_{0}=1, \theta_{1}=1, C_{0}=1, C_{1}=1 & \text { at } y=0 \\
u_{0} \rightarrow u_{1} \rightarrow 1, \theta_{0} \rightarrow 0, \theta_{1} \rightarrow 0, C_{0} \rightarrow 0, C_{1} \rightarrow 0 & \text { as } y \rightarrow \infty
\end{array}
$$

Finally solving equation (22) to equation (27), we obtain the analytical solutions of equation (29) to equation (34), satisfying the above boundary conditions (28) are as follows

$$
u_{0}=1+J_{1} e^{m_{2} y}+J_{2} e^{m_{6} y}+J_{3} e^{m_{2} y}+J_{4} e^{m_{10} y}
$$

In view of the above solutions the velocity, temperature and concentration distributions in the boundary layer 


$$
C(y, t)=B_{1} e^{m_{2} y}+B_{2} e^{m_{6} y}+\varepsilon e^{n t}\left(B_{3} e^{m_{6} y}+B_{4} e^{m_{2} y}+B_{5} e^{m_{8} y}+D_{3} e^{m_{2} y}+D_{4} e^{m_{4} y}\right)
$$

It is now important to calculate the physical quantities of primary interest, which are the local wall shear stress, the local surface heat and mass flux. Using the velocity field in

$$
C_{f}=\left(\frac{\partial u}{\partial y}\right)_{y=0}=J_{1}+J_{2}+J_{3}+J_{4}+\varepsilon e^{n t}\left(J_{6}+J_{7}+J_{8}+J_{9}+J_{10}+J_{11}+J_{12}+J_{13}+J_{14}+J_{15}+J_{16}+J_{17}\right)
$$

The dimensionless local surface heat flux i.e., Nusselt number $(N u)$ is can be written as

$$
N u=\frac{q_{w}^{*}}{k\left(T_{w}^{*}-T_{\infty}^{*}\right)} \Rightarrow N u R e_{x}=-\left(1+\frac{4 R}{3}\right)\left(\frac{\partial \theta}{\partial y}\right)_{y=0}=-\left(1+\frac{4 R}{3}\right)\left[m_{2}+\varepsilon e^{n t}\left(m_{2} D_{1}+m_{4} D_{2}\right)\right]
$$

Where $q_{w}^{*}=-\frac{k\left(T_{w}^{*}-T_{\infty}^{*}\right) V_{0}}{v}\left(1+\frac{4 R}{3}\right)\left(\frac{\partial \theta}{\partial y}\right)_{y=0}$ and $R e_{x}$ is the Reynolds number.

The Sherwood number is given by

$$
S h=\left(\frac{\partial C}{\partial y}\right)_{y=0}=m_{2} B_{1}+m_{6} B_{2}+\varepsilon e^{n t}\left(m_{6} B_{3}+m_{2} B_{4}+m_{8} B_{5}\right)
$$

\section{Results and Discussion}

In order to get a clear insight of the physical problem, numerical results are displayed with the help of graphs. This enables us to carry out the numerical calculations for the distribution of the velocity, temperature, concentration, Nusselt number and Sherwood number across the boundary layer for various values of the flow parameters. To be realistic, the value of Schmidt number $(S c)$ are chosen for $S c=0.22,0.30, .065$ and 0.78 . The value of prandtl number $(P r)$ are chosen for Helium $P r=0.71$ at $366 \mathrm{~K}$ temperature, for water $P \mathrm{r}=7.02$ at $20^{\circ} \mathrm{C}$, for stream $\operatorname{Pr}=1.00$, for Ammonia $P r=2.00$ at $40^{\circ} \mathrm{C}$. Grashof number for heat transfer is chosen to be $G r=1.0$ Modified Grashof number for mass transfer is chosen to be $G c=2.0$. The velocity profiles $u$ for different values of the above parameter are illustrated in Figure 2 to Figure 9, the temperature profiles for different values of the above parameter are displayed in Figure 10 to Figure 12 and the concentration profiles for different values of the above parameter are shown in Figure 13 to Figure 17. Nusselt number for different values of the above parameter is displayed in Figure 18. Skin friction for different values of the above parameter is let on in Figure 19. Finally the Sherwood number for different values of the above parameter is illustrated in Figure 20.

The velocity profile for different values of Grashof number $(G r)$ are described in Figure 2. From this figure it is observed that an increasing in $G r$ leads to increase in the values of velocity. Here the Grashof number leads free convection currents. If $G r=0$ then it represents the absence of free convection currents. $G r>0$ means heating of the fluid of cooling of the boundary surface and $G r<0$ means cooling of the fluid of heating of the boundary surface. From the Figure 2 the velocity attains its highest value at $y=1.0$.

The velocity profile for different values of modified Grashof number for mass transfer $(G c)$ are described in
Figure 3. It is observed that an increasing in $G c$ leads to increase in the values of velocity. The curves evince that the peak value of velocity increases rapidly near the wall of the porous plate as modified Grashof number for mass transfer $(G c)$ increases. At $y=1.2$ the velocity is maximum for $G c=4.0$.

Figure 4 evince the velocity for different values of the permeability $(K)$.It is clear that the peak value of the velocity tends to increases as permeability $(K)$ increases. At $y=1.25$ (approximately) the velocity is highest.

For different values of the magnetic field parameters $(M)$, the velocity profiles are plotted in Figure 5 . It is obvious that the effect of increasing values of $M$ results in decreasing velocity distribution across the boundary layer. At $y=1.0$ (approximately) the velocity is highest.

Figure 6 exhibit the velocity profiles across the boundary layer for different values of Prandtl number $(P r)$. It is obvious that the effect of increasing values of Prandtl number $(\mathrm{Pr})$ results in decreasing the velocity. Typical variations of the temperature profiles along the span wise coordinate $y$ are shown in Figure 12 for different values of Prandtl number $(P r)$. The result shows that for an increasing value of Prandtl number leads an increasing the temperature profiles of thermal boundary layer thickness and constant temperature distribution across the boundary layer. Figure 15 describes the concentration profiles for various values of Prandtl number. It is noticed that the concentration profiles increases near the porous plate and decreases far away from the porous plate as Prandtl number increases.

The effect of Soret number $\left(S_{0}\right)$ on the velocity profiles is shown in Figure 7. From this figure we see that the velocity profiles increase with an increasing of $S_{0}$ from which we conclude that the fluid velocity rises due to greater thermal diffusion. Figure 17 represents the concentration profiles for different values of Soret number. From the figure we observe that the concentration profiles increase with an increasing value of Soret number. The effect of Soret number $\left(S_{0}\right)$ on the Sherwood number $(S h)$ shown in the Figure 20. It is noticed that for an increase of $S_{0}$ the Sherwood number increases.

The effect of radiation parameter $(R)$ on the temperature 
profiles is shown in Figure 10. From this figure we observe that the temperature profiles decreases for an increasing value of $R$, with an increasing in the thermal boundary layer thickness. Figure 14 represents the effect of radiation parameter on concentration profiles. Here we find that as the value of $R$ increases the concentration increases, with an increasing in the flow boundary layer thickness. Figure.19 depicts the effect of the radiation parameter $(R)$ on skin friction coefficient $C_{f}$ versus $G r$. It is observed that as $R$ increase, the skin friction coefficient increases. Figure 18 is displayed the effect of $R$ on Nusselt number. It is observed that as the radiation parameter increases, the Nusselt number $(\mathrm{Nu})$ decreases.

For different values of the Schmidt number $(S c)$, the velocity profiles are plotted in Figure 8. It is obvious that the effect of increasing values of $S c$ parameters results in decreasing velocity distribution across the boundary layer. The concentrations profiles are plotted in Figure 16 for different values of the Schmidt number $(S c)$. It is observed that the effect for increasing values of $S c$ results in decreasing concentrations distribution across the boundary layer and all curves meet the $y$ axis. The curve is too much high at the wall.

Figure 9 and Figure 13 evince the effect of heat source parameter $(Q)$ on the velocity and concentration profiles. From the Figure 9 and Figure 13, we see that the heat is generated the buoyancy force increases which induces the coincidence in the velocity curves and the flow rate to increase giving rise to the concentration profiles. Figure 11 shows the temperature profiles for different values of the heat source parameters $(Q)$. From the Figure 11 we see that the temperature profiles decreases with an increasing of heat source parameters $(Q)$.

Comparing with the previous work of Bhavana and Kesavaiah [10], we have seen that there is only different effect of permeability parameter on the velocity profile as well as radiation parameter effect on temperature profiles. There is an excellent agreement on effect of all other flow parameters of the pervious works.

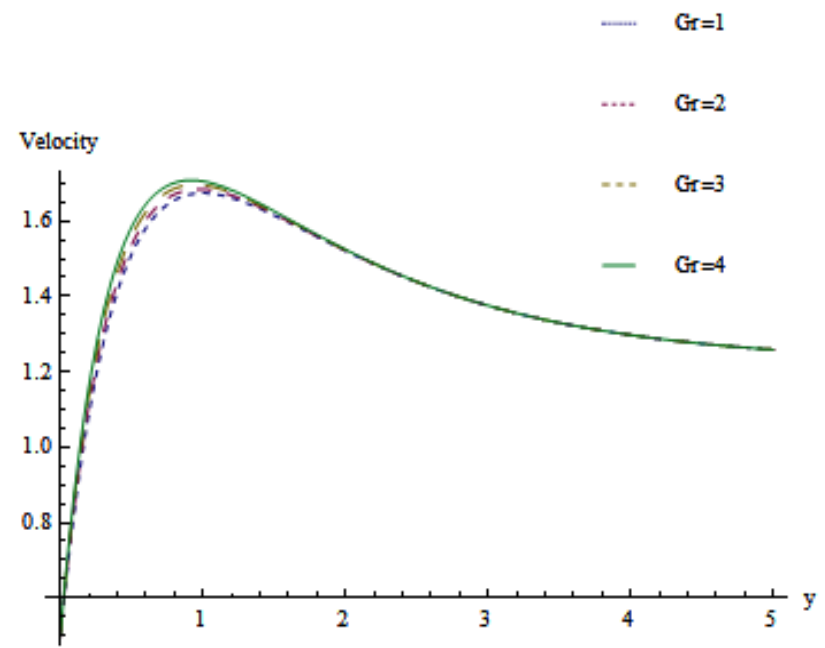

Figure 2. Velocity profiles with $\operatorname{Pr}=7, R=1, A=0.5, S c=0.65, M=1, Q=1$, $\varepsilon=0.2, G c=2, n=0.1, t=1, K=0.5, n=0.1, S_{0}=1$ and $U p=0.5$ for different values of Gr against $y$.

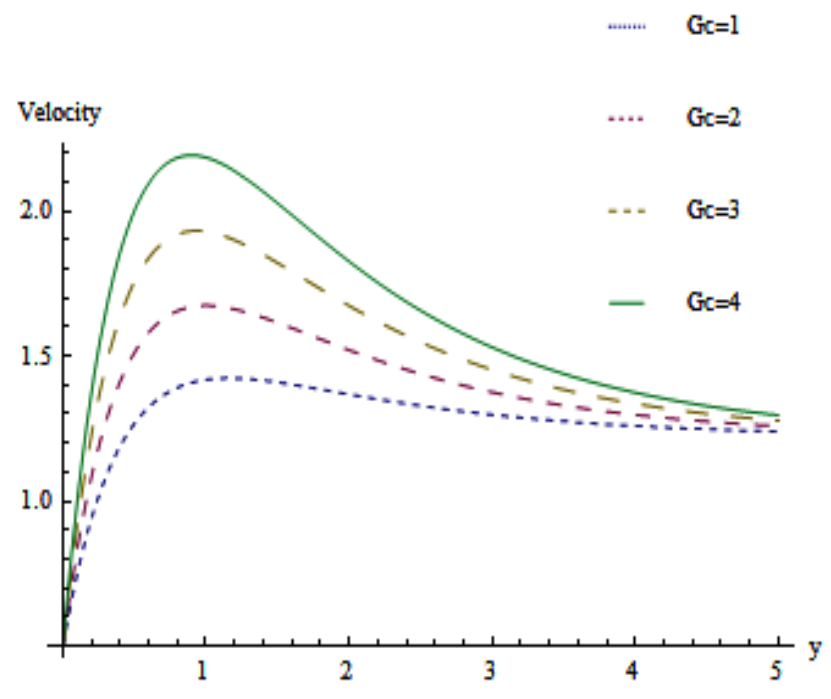

Figure 3. Velocity profiles with $\operatorname{Pr}=7, M=1, S c=0.65, G r=1, t=1$, $K=0.5, \varepsilon=0.2, R=1, A=0.5, Q=1, n=0.1, S_{0}=1$ and $U p=0.5$ for different values of Gc against $y$.

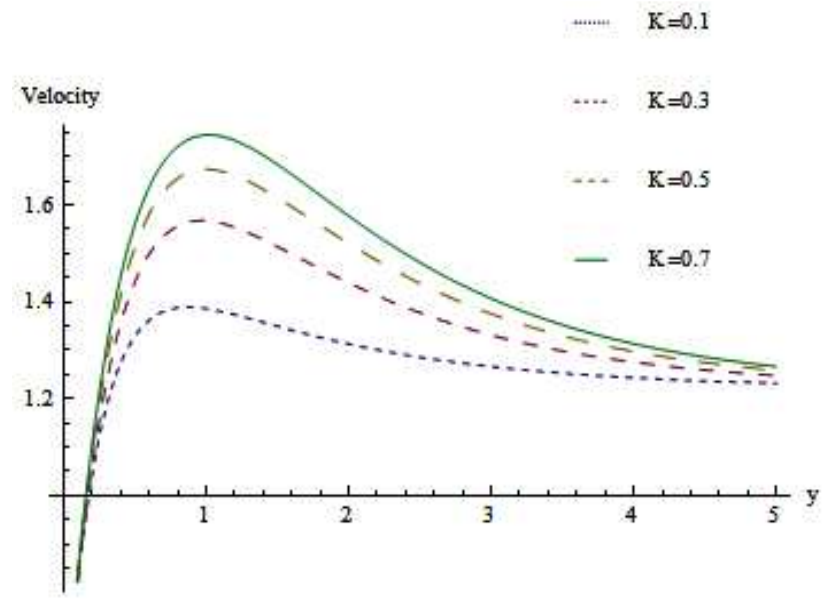

Figure 4. Velocity profiles with $M=1, S c=0.65, G c=2, G r=1, t=1, P r=7$, $U p=0.5, S_{0}=1, n=0.1, Q=1, A=0.5, \varepsilon=0.2$ and $R=1$ for different values of $K$ against $y$.

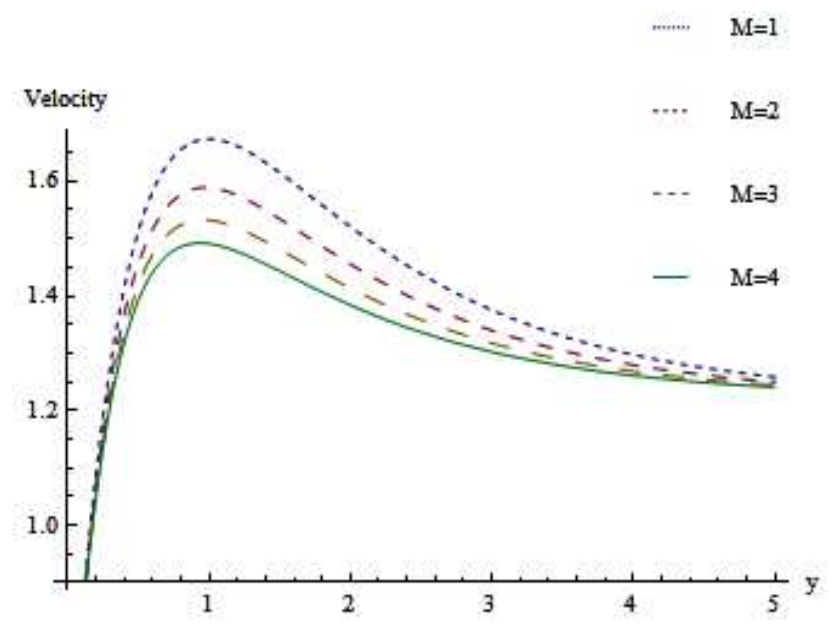

Figure 5. Velocity profiles with $S c=0.65, G c=2, G r=1, t=1, K=0.5, \operatorname{Pr}=7$, $n=0.1, Q=1, A=0.5, \varepsilon=0.2, R=1, U p=0.5$ and $S_{0}=1$ for different values of $M$ against $y$. 


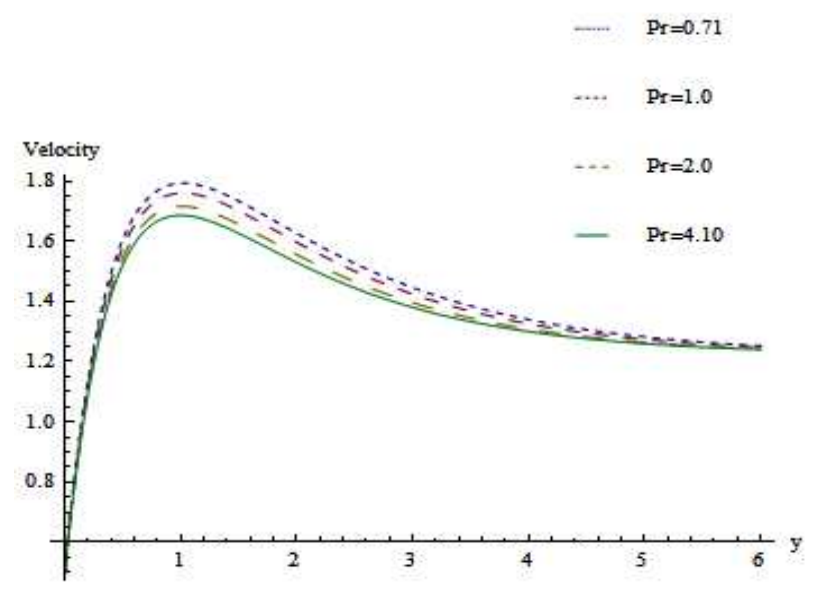

Figure 6. Velocity profiles with $M=0.5, S c=0.65, G c=2, G r=1, \quad t=0.01$, $K=0.5, R=1, A=0.5 Q=.01, n=0.1, S_{0}=1, U p=0.5$ and $\varepsilon=0.2$ for different values of Pr against $y$.

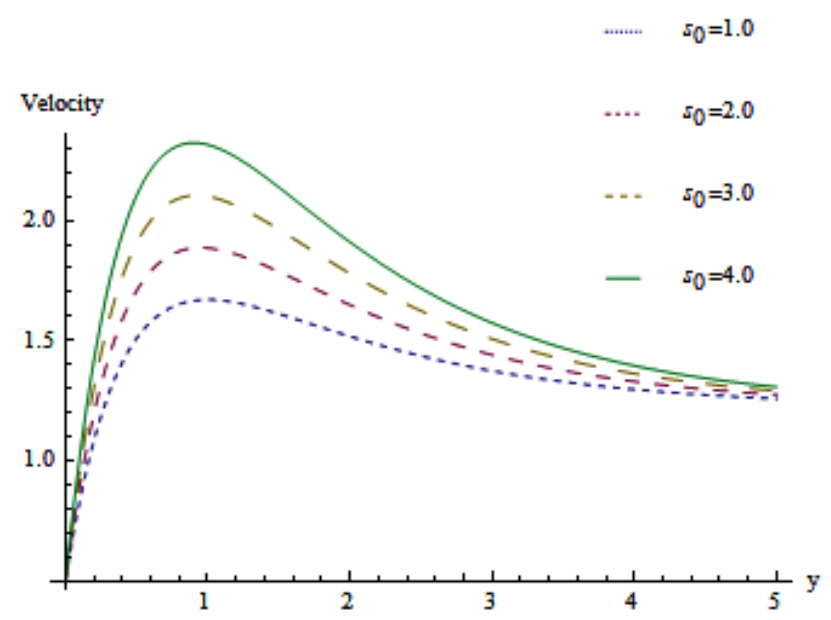

Figure 7. Velocity profiles with $M=1, S c=0.65, G c=2, G r=1, t=1, K=0.5$, $\operatorname{Pr}=7, n=0.1, Q=1, A=0.5, \varepsilon=0.2, R=1$ and $U p=0.5$ for different values of $S_{0}$ against $y$.

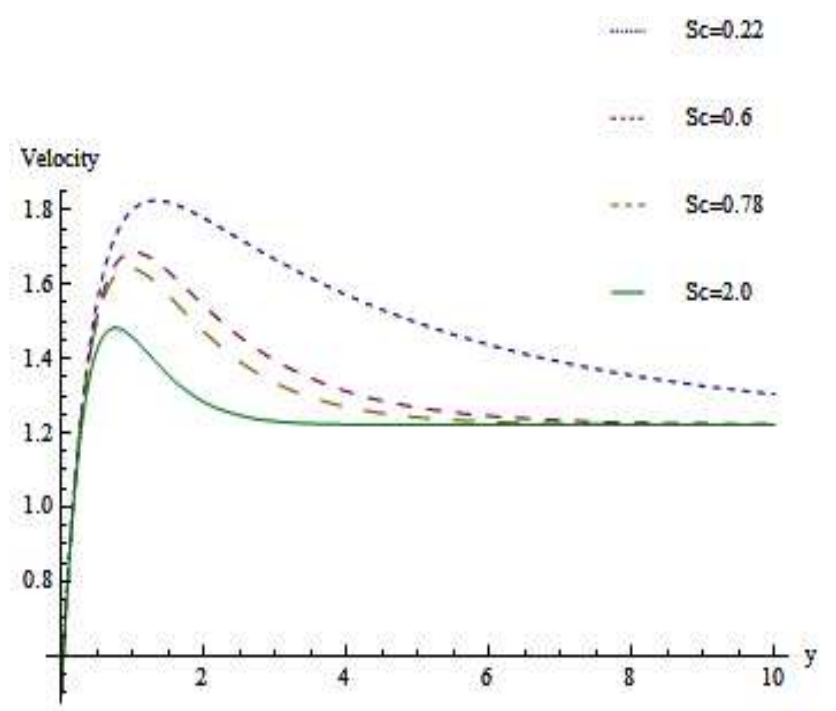

Figure 8. Velocity profiles with $M=1, G c=2, G r=1, t=1, K=0.5, \operatorname{Pr}=7$, $n=0.1, Q=1, A=0.5, \varepsilon=0.2, R=1, U p=0.5$ and $S_{0}=1$ for different values of $S c$ against $y$.

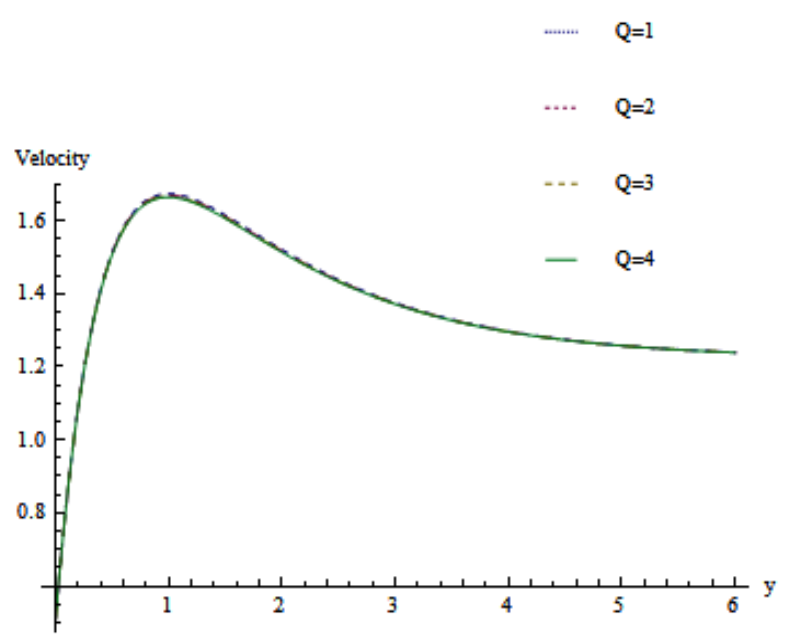

Figure 9. Velocity profiles with $\operatorname{Pr}=7, M=1, S c=0.65, G c=2, G r=1, t=1$, $\varepsilon=0.2, K=0.2, R=1, A=0.5, n=0.1, U p=0.5$, and $S_{0}=1$ for different values of $Q$ against $y$.

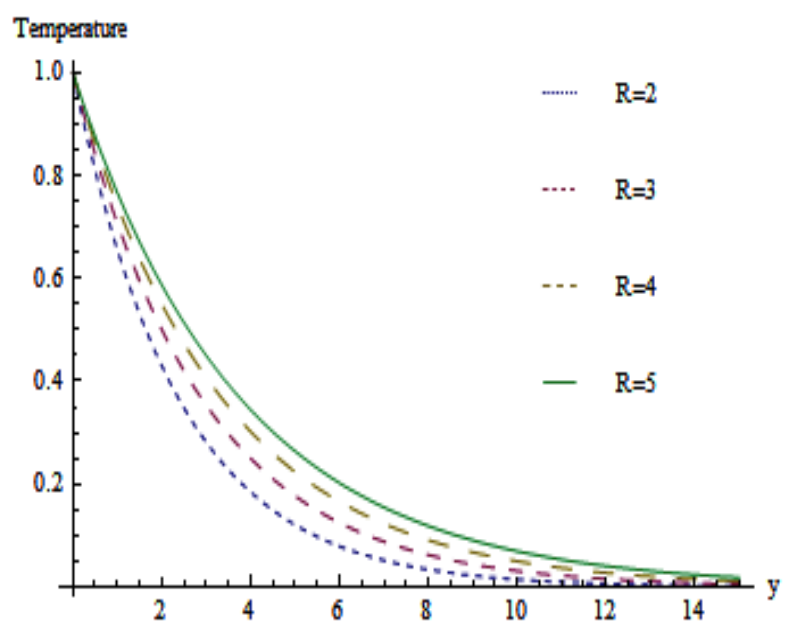

Figure 10. Temperature profile with $\operatorname{Pr}=7, M=1, S c=0.65, G c=2, G r=1, t=1$, $K=0.2, \quad A=0.5, n=0.1, \quad U p=0.5, \varepsilon=0.02 \quad P r=0.71$ and $M=1$ for different values of $R$ against $y$.

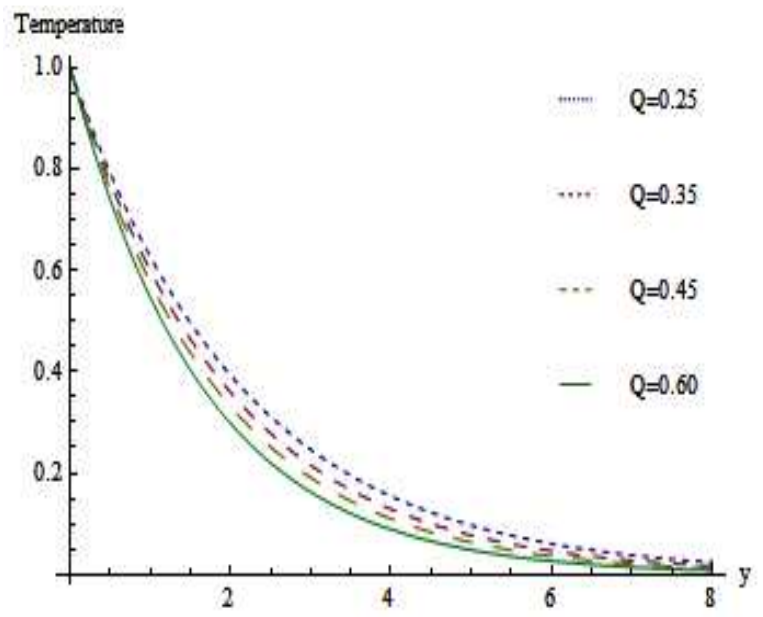

Figure 11. Temperature profile with $\operatorname{Pr}=0.71, M=1, S c=0.65, G c=2, G r=1$, $t=1, K=0.5, \varepsilon=0.02, R=1, A=0.5, n=1$ and $U p=0.5$ for different values of $Q$ against $y$. 


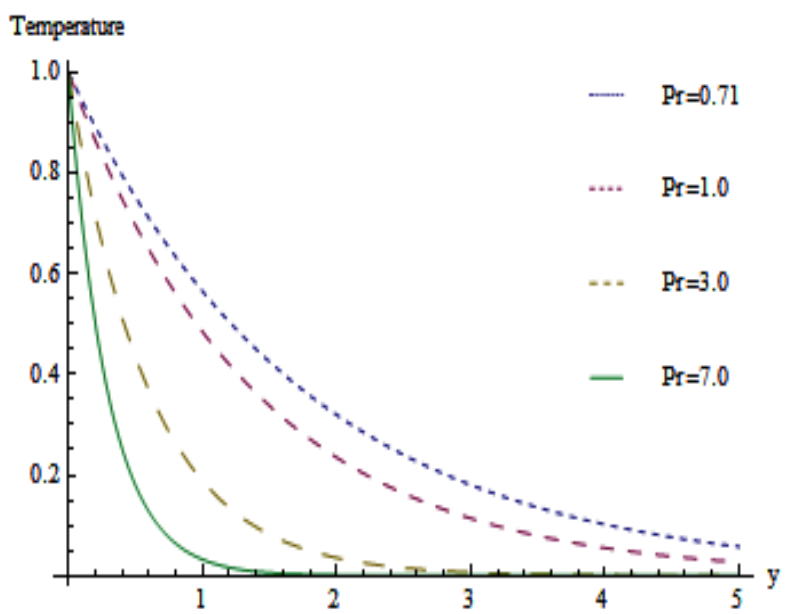

Figure 12. Temperature profile with $P r=0.71, M=1, S c=0.65, G c=2, G r=1$ $t=1, K=0.5, \varepsilon=0.02, R=1, A=0.5, n=1, S_{0}=1$ and $U p=0.5$ for different values of Pr against $y$.

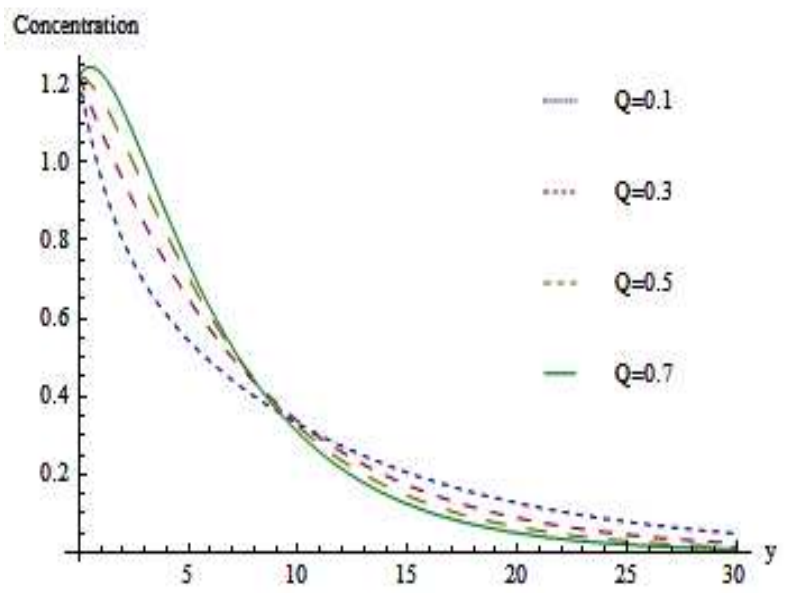

Figure 13. Concentration profile with $G c=2, \operatorname{Pr}=0.71, M=1, S c=0.65, G r=1$, $t=1, K=0.5, \varepsilon=0.02, R=1, A=0.5, n=1, S_{0}=1$ and $U p=0.5$ for different values of $Q$ against $y$.

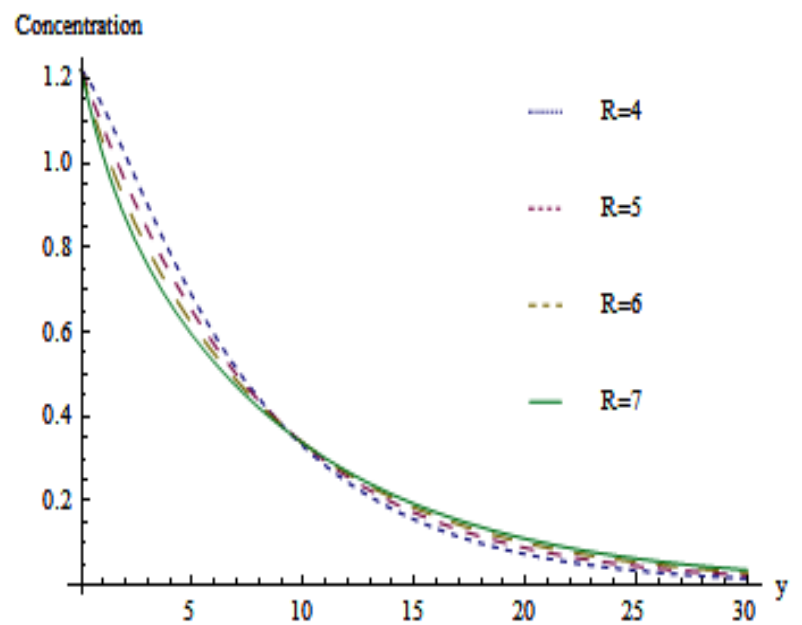

Figure 14. Concentration profiles with $G c=2, \operatorname{Pr}=0.71, M=1, S c=0.65$, $G r=1, t=1, K=0.5, \varepsilon=0.02, A=0.5, Q=0.5, n=1, S_{0}=1$ and $U p=0.5$ for different values of $R$ against $y$.

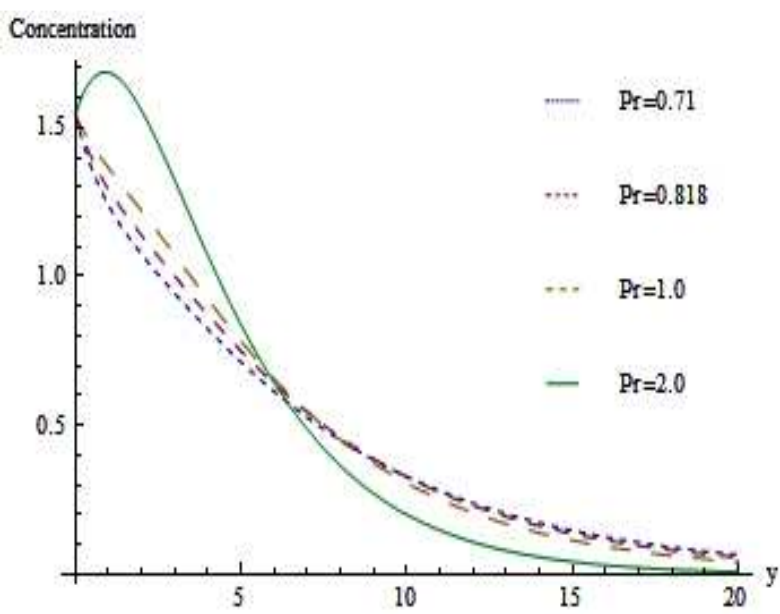

Figure 15. Concentration profiles with $G c=2, M=1, S c=0.65, G r=1, t=1$, $K=0.5, \varepsilon=0.02, A=0.5, Q=0.5, n=1, S_{0}=1, U p=0.5$ and $R=3.5$ for different values of Pr against $y$.

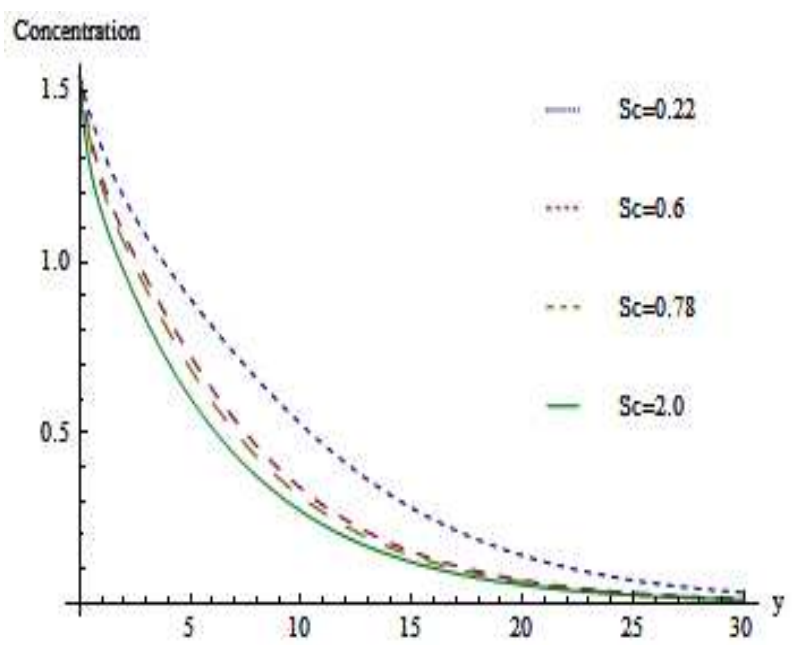

Figure 16. Concentration profiles with $G c=2, M=1, S c=0.65, G r=1, t=1$, $K=0.5, \varepsilon=0.2, A=0.6, Q=0.5, n=0.1, S_{0}=1, U p=0.5, R=0.1$ and $\operatorname{Pr}=0.71$ for different values of $S c$ against $y$.

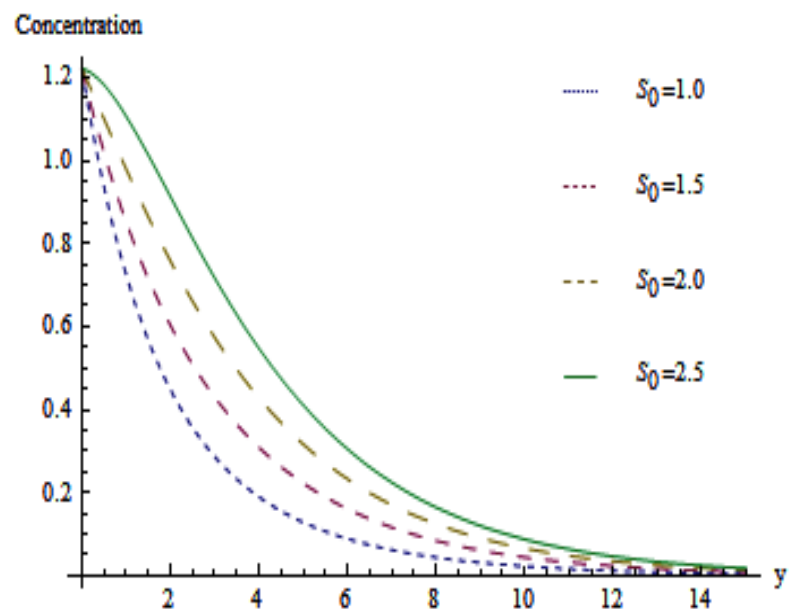

Figure 17. Concentration profiles with $G c=2, M=1, S c=0.65, G r=1, t=1$, $K=0.5, \varepsilon=0.02, A=0.5, Q=0.5, n=1, P r=0.71, U p=0$ and, $R=3$ for different values of $S_{0}$ against $y$. 


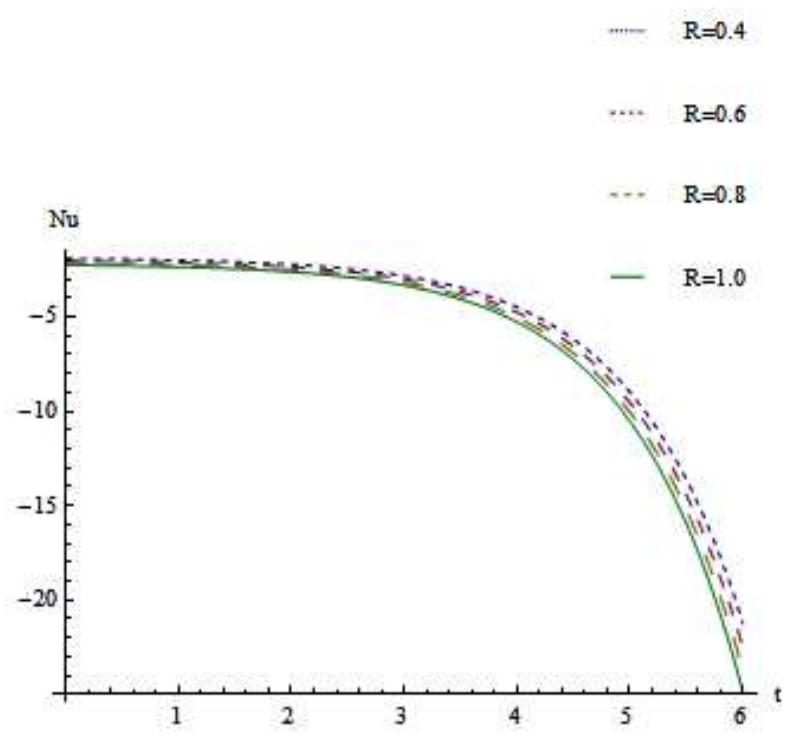

Figure 18. Nusselt number profiles with $S c=0.65, K=0.5, \varepsilon=0.02, A=0.5$, $Q=2.0$ and $P r=0.71$ for different values of $R$ against $t$.

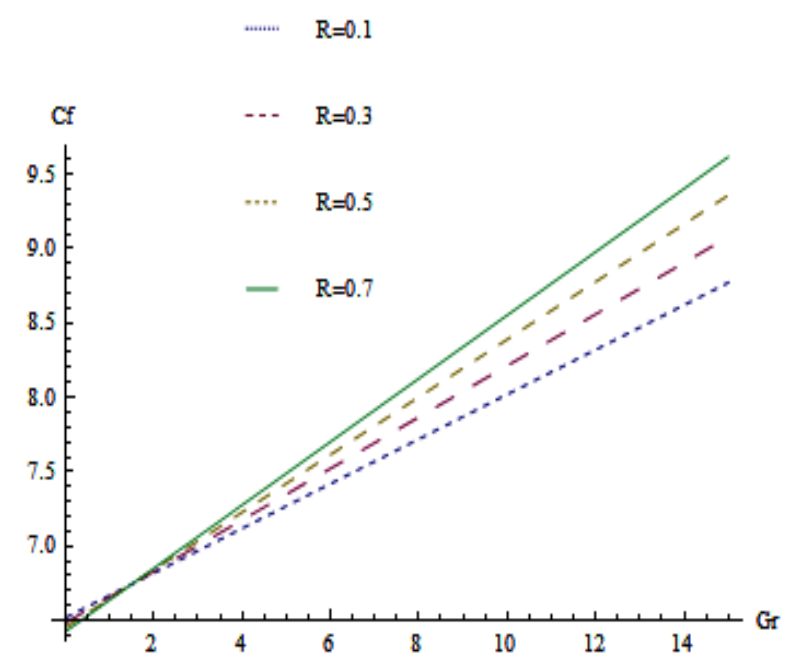

Figure 19. Skin friction profiles with $G c=5, M=1, S c=0.65, K=0.6, \varepsilon=0.2$, $Q=1.0, n=0.1 S_{0}=1, U p=0.5, R=0.1$ and $P r=7.0$ for different values of $R$ against $\mathrm{Gr}$.

\section{Appendix}

$$
\begin{aligned}
& m_{2}=-\frac{1+\sqrt{1+4 Q \beta_{1}}}{2 \beta_{1}} \\
& m_{4}=-\frac{1+\sqrt{1+4(n+Q) \beta_{1}}}{2 \beta_{1}} \\
& \mathrm{~m}_{6}=-S c \\
& m_{8}=-\frac{S c+\sqrt{(S c)^{2}+4 n S c}}{2} \\
& m_{10}=-\frac{1+\sqrt{1+4 N}}{2}
\end{aligned}
$$

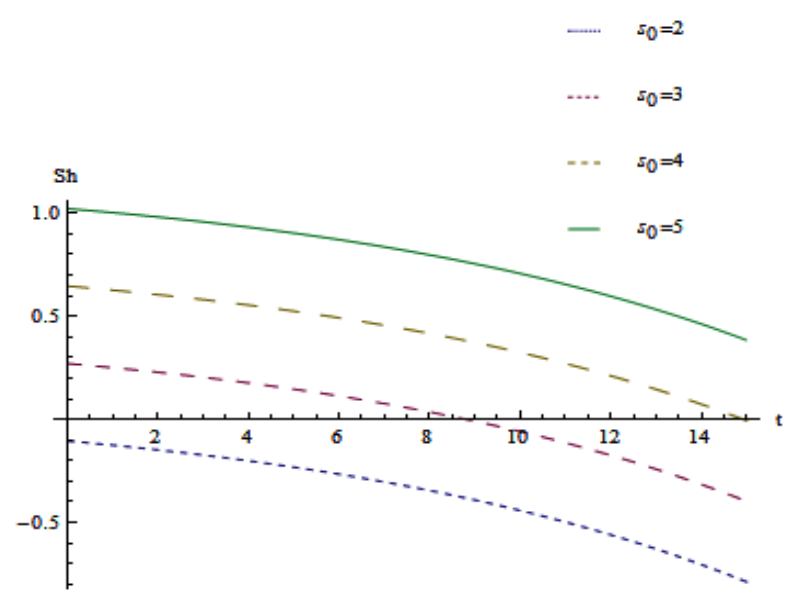

Figure 20. Sherwood number profiles $S c=0.65, \varepsilon=0.2, A=0.6, Q=0.5$, $n=0.1,=1, U p=0.5, R=1.0$ and $P r=0.71$ for different values of $S_{0}$ versus $t$.

\section{Conclusions}

In the present research work, the boundary layer equations become non-dimensional by using non-dimensional quantities. The non dimensional boundary layer equations are non linear partial differential equations. These equations are solved by using perturbation technique. The following conclusions are set out through the overall observations.

1) Velocity increases with an increase of Grashof number $(G r)$, modified Grashof number for mass transfer $(G c)$, Permeability $(K)$ and Soret number $\left(S_{0}\right)$. Whereas velocity decreases with an increase of Prandtl number $(P r)$, magnetic field parameter $(M)$ and Schmidt number $(S c)$. There is no effect of heat source parameter $(Q)$ on the velocity profiles.

2) The temperature and the skin friction increase with an increasing value of radiation parameter $(R)$ also temperature decreases with the increasing value Prandtl number $(P r)$ and, heat source parameter $(Q)$.

3) The Nusselt number $(\mathrm{Nu})$ decreases with an increasing value of radiation parameter $(R)$ and the Sherwood number increases with an increasing value of Soret number $\left(S_{0}\right)$.

$$
\begin{aligned}
& J_{1}=\frac{-G r}{m_{2}{ }^{2}+m_{2}-N} \\
& J_{2}=\frac{-G c B_{2}}{m_{6}{ }^{2}+m_{6}-N} \\
& J_{3}=\frac{-G c B_{1}}{m_{2}{ }^{2}+m_{2}-N} \\
& J_{4}=\left(U_{p}-1-J_{1}-J_{2}-J_{3}\right) \\
& J_{6}=-\frac{A J_{4} m_{10}}{m_{10}{ }^{2}+m_{10}-(N+n)}
\end{aligned}
$$




$$
\begin{aligned}
& m_{12}=-\frac{1+\sqrt{1+4(N+n)}}{2} \\
& \beta_{1}=\frac{(3+4 R)}{3 \operatorname{Pr}} \\
& D_{1}=\frac{-A m_{2}}{\beta_{1} m_{2}^{2}+m_{2}-(n+Q)} \\
& D_{2}=\left(1-D_{1}\right) \\
& D_{3}=\frac{-S c S_{0} m_{2}^{2} D_{1}}{m_{2}^{2}+S c m_{2}-n S c} \\
& D_{4}=\frac{-S c S_{0} m_{4}^{2} D_{2}}{m_{4}^{2}+S c m_{4}-n S c} \\
& B_{1}=\frac{-S c S_{0} m_{2}}{m_{2}+S c} \\
& B_{2}=\left(1-B_{1}\right) \\
& B_{5}=\left(1-B_{3}-B_{4}-D_{3}-D_{4}\right) \\
& B_{4}=\frac{-A S c m_{6} B_{2}}{m_{6}^{2}+S c m_{6}-n S c} \\
& m_{2}^{2}+S c m_{2}-n S c
\end{aligned}
$$

\section{References}

[1] A. K. Singh, "MHD free convective flow past an accelerated vertical porous plate by finite difference method." Astrophys. Space Sci. 94, 395-400, 1983.

[2] A. Raptis, G.T Zivnidis, N. Kafousis, "Free convection and mass transfer flow through porous medium bounded by an infinite vertical limiting surface with constant suction." Letters in heat and mass transfer, 8, 5,417-424, 1981.

[3] M. D. Abdus-sattar and M. D. Hamid Kalim, "Unsteady free convection interaction with thermal radiation in a boundary layer flow past a vertical porous plate." J. Math. Phys. Sci. 30, pp. 25-37, 1996.

[4] S.S. Das, S.K. Sahoo and G.C. Dash, "Numerical solution of mass transfer effects on unsteady flow past an accelerated vertical porous plate with suction." Bull. Malays. Math. Sci. Soc., 2, 29, (1), 33-42, 2006.

[5] S. S. Das, A. Satapathy, J. K. Das, J. P. Panda, "Mass transfer effects on MHD flow and heat transfer past a vertical porous plate through porous medium under oscillatory suction and heat source." International journal of heat and mass transfer, volume 52, Issues 25-26, December 2009, Pages 5962-5969.

[6] B. K. Jah, "Applied magnetic field on transient convective flow in a vertical channel."J. Pure Appl. Math. 29, 441-445, 1998.

$$
\begin{aligned}
J_{7} & =-\frac{A J_{1} m_{2}}{m_{2}^{2}+m_{2}-(N+n)} \\
J_{8} & =-\frac{A J_{2} m_{6}}{m_{6}^{2}+m_{6}-(N+n)} \\
J_{9} & =-\frac{A J_{3} m_{2}}{m_{2}^{2}+m_{2}-(N+n)} \\
J_{10} & =-\frac{G r D_{2}}{m_{4}^{2}+m_{4}-(N+n)} \\
J_{11} & =-\frac{G r D_{1}}{m_{2}^{2}+m_{2}-(N+n)} \\
J_{12} & =-\frac{G c B_{5}}{m_{4}^{2}+m_{4}-(N+n)} \\
J_{13} & =-\frac{G c B_{3}}{m_{6}^{2}+m_{6}-(N+n)} \\
J_{17} & =-\left(1+J_{7}+J_{8}+J_{9}+J_{6}+J_{11}+J_{10}\right. \\
J_{16} & =-\frac{G c B_{4}}{m_{4}^{2}+D-(N+n)} \\
J_{15} & =-\frac{G c D_{3}}{m_{2}^{2}+m_{2}-(N+n)} \\
J_{13}+J_{14}+J_{12}+(N+n) & \left.J_{16}\right) \\
J_{15} &
\end{aligned}
$$

[7] Y. J. Kim, "Unsteady MHD convective heat transfer past a semi-infinite vertical porous moving plate with variable suction." Int. J. Engg.Sci.38, 833-445, 2000.

[8] V. M. Soundalgekar, B. S. Jaisawal, A. G. Uplekar and H. S. Takhar, "The transient free convection flow of a viscous dissipative fluid past a semi-infinite vertical plate." J. Appl. Mech. Engng.4, 203-218, 1999.

[9] R. A. Mohameda, Ibrahim A. Abbasb, S.M. Abo-Dahaba, "Finite element analysis of hydromagnetic flow and heat transfer of a heat generation fluid over a surface embedded in a non-darcian porous medium in the presence of chemical reaction." Science and Numerical Simulation. 14, 4, 13851395, 2009.

[10] M Bhavana, D Chenna Kesavaish "The Soret Effect on free convective unsteady MHD Flow over a vertical plate with Heat Source." Int. Journal of Innovative Research in science and technology, Vol. 2, May 2013.

[11] M. A Abd EL-Naby, E M E. El-Barbary and N.Y. Abdelazem "Finite difference solution of radiation effects on MHD unsteady free-convection flow on vertical porous plate", Appl. Math. Comput.151 (2), pp. 327 -346, 2004.

[12] M.M Alam,. M. S Rahman, "Dufour and soret effects on mixed convection flow past a vertical porous flat plate with variable suction", Nonlinear analysis: modeling and control, Vol. 11, No. 1, pp. 3-12, 2006. 
[13] O Beg Anwa., A.Y. Bakier and V. R. Prasad, "Numerical study of free convection magneto hydrodynamic heat and mass transfer from a stretching surface to a saturated porous medium with Soret and Dufour effects", Computational Material Science, 46, pp. 57-65, 2009.

[14] F. M Hady, R. A. Mohamed and A. Mahdy A "MHD free convection flow along a vertical wavy surface with heat generation or absorption effect", Int. Comm. Heat Mass Transfer, 33, pp. 1253-1263, 2006.

[15] O. D Makinde, "Free-convection flow with thermal radiation and mass transfer past a moving vertical porous plate", Int. Comm. Heat Mass Transfer, 32, pp. 1411- 1419, 2005.
[16] I. U. Mbeledogu, A. R. C. Amakiri and A. Ogulu, "Unsteady MHD free convection flow of a compressible fluid past a moving vertical plate in the presence of radioactive heat transfer", Int. J. of Heat and Mass Transfer, 50, pp. 16681674, 2007.

[17] S. F. Ahmmed, S. Mondal and A. Ray, "Numerical studies on MHD free convection and mass transfer flow past a vertical flat plate." IOSR Journal of Engineering (IOSRJEN). ISSN 2250-3021, vol. 3, Issue 5, pp 41-47, May. 2013. 